\section{ALGUNAS CUESTIONES METODOLÓGICAS EN EL ANÁLISIS ECONÓMICO}

\author{
SOME METHODOLOGICAL ISSUES \\ IN ECONOMIC ANALYSIS
}

\author{
Víctor A. Beker
}

\section{RESUMEN}

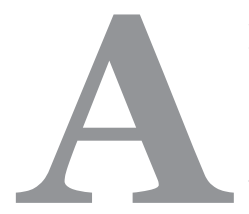

partir de la crisis financiera de 2007/2008 la teoría económica predominante se encuentra en estado de crisis. La mayoría de sus economistas no sólo no previeron la profundidad de la crisis sino que ni siquiera consideraban que algo así fuera posible. Este estado de cosas ha resucitado el interés por las cuestiones metodológicas del análisis económico. En este trabajo se revisan algunas cuestiones destacadas en materia de metodología económica. Ellas son: la diferencia entre la ciencia económica y las ciencias naturales; cómo opera la refutación en economía; las posibilidades de predecir en esta ciencia; el uso de la matemática; la relación entre economía positiva y economía normativa y se explora la posibilidad de utilizar el enfoque de complejidad en economía.

Palabras clave: metodología, refutación, predicción, matemática, complejidad.

\section{ABSTRACT}

From the financial crisis of 2007/2008 mainstream economic theory is in crisis. Most mainstream economists not only did not anticipate the depth of the crisis but not even considered that something like that was possible. This state of things has reawakened the interest in methodological issues in economic analysis. In this article some outstanding questions in economic methodology are analyzed. They are: the difference between economic science and natural sciences; how refutation works in economics;

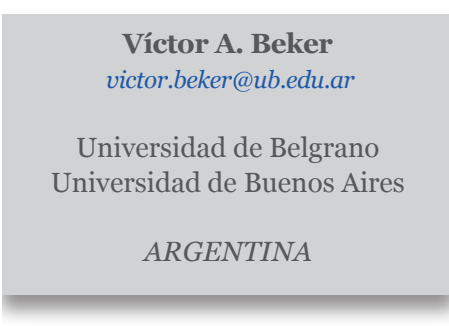

Víctor A. Beker

Universidad de Belgrano

Universidad de Buenos Aires

ARGENTINA 
the possibilities of predicting in this science; the use of the mathematics; the relation between positive and normative economics and, finally, explores the possibility of using the complexity approach in economics.

Keywords: Actions. types of actions, actions with the right to veto.

\section{1.- INTRODUCCIÓN}

El objetivo de este artículo es revisar algunas cuestiones destacadas en materia de metodología económica.

A partir de la crisis financiera de 2007/2008 la teoría económica predominante se encuentra en estado de crisis. La ortodoxia no estaba preparada para lidiar con semejante fenómeno. La mayoría de sus economistas no sólo no previeron la profundidad de la crisis sino que ni siquiera consideraban que algo así fuera posible. Para ellos, el apartamiento del equilibrio sólo podía ser temporario: no sólo suponían que la economía tiende siempre al equilibrio sino, además, que el mismo es estable. Si bien es cierto que la Gran Recesión no se convirtió en otra Gran Depresión como la de los años 1930 ello fue gracias al uso de políticas heterodoxas, fundamentalmente de cuño keynesiano.

Este estado de cosas ha resucitado el interés por las cuestiones metodológicas del análisis económico.

El artículo aborda en la sección 2 la contradicción entre lo que los economistas pregonan desde el punto de vista metodológico y lo que practican; la sección 3 está dedicada a analizar el tema de la refutación en economía; la sección 4 aborda el tema de la predicción; en la siguiente se analiza el uso de la matemática en economía; en la sección 6 se discute la relación entre economía positiva y normativa; en la 7, se introduce el enfoque de complejidad. Finalmente, la sección 8 está dedicada a las conclusiones.

\section{2.- LA ESQUIZOFRENIA DE LOS ECONOMISTAS}

Al igual que el resto de las ciencias, la economía formula teorías falseables. Sin embargo, la peculiaridad que la distingue de, por ejemplo, las ciencias naturales, es que sus teorías, en la mayoría de los casos, no pueden ser falseadas.

La teoría económica, como sucedió con otras disciplinas, se desarrolló durante muchos años sin que sus estudiosos sintieran la necesidad de interrogarse a sí mismos respecto a las 
cuestiones metodológicas. Sólo de vez en cuando algunos de sus practicantes hicieron alguna incursión en esa zona. Senior, Mill, Cairnes, Menger, Keynes (padre), Robbins, Hutchison y Machlup forman una lista no exhaustiva de quienes estaban más interesados en el tema de cómo los economistas hacen (o deberían hacer) lo que hacen. Si hubo un trabajo influyente sobre cuestiones metodológicas sin duda alguna fue el de Friedman (1953). El mismo se convirtió en un destacado punto de referencia en el debate sobre metodología económica tanto para sus seguidores como para sus críticos.

Un cambio significativo en materia de metodología económica tuvo lugar en la década de 1980, reflejando en parte los debates paralelos que tuvieron lugar en el área de la filosofía de la ciencia. La década abrió, precisamente, con Blaug $(1980,1992)$, que intentó ubicar a Popper en el centro del debate metodológico en economía. Durante la década de 1980 la literatura sobre metodología económica creció exponencialmente. Los autores venían tanto del campo de la economía como del de la filosofía de la ciencia. Entre estos últimos, Hausman (1992) llamó la atención sobre lo que él llamó la esquizofrenia metodológica de la economía contemporánea, refiriéndose al hecho de que generalmente los pronunciamientos metodológicos y la práctica económica se contradicen entre sí.

Hausman señala que los economistas que adhieren al falsacionismo -ya sea en su versión popperiana o lakatosiana- y escriben sobre cuestiones metodológicas, describen su actividad como si ellos siguieran estrictamente ese enfoque. Los economistas formularían teorías, deducirían de ellas las predicciones y someterían a prueba dichas predicciones. Si las predicciones no son correctas, la teoría sería desestimada o reemplazada por una teoría alternativa con un mayor grado de verosimilitud. Hausman plantea que la práctica de los economistas tiene poco que ver con esta descripción. Hausman sostiene que lo que hacen los economistas (independientemente de lo que puedan decir en discusiones metodológicas) es aplicar las siguientes reglas (Hausman 1992, p.222):

1. Formular generalizaciones acerca de la operación de factores causales relevantes.

2. Deducir de tales generalizaciones y de las condiciones iniciales, simplificaciones, etc., predicciones concernientes a fenómenos relevantes.

3. Contrastar tales predicciones.

4. Si las predicciones son correctas, considerar todo el conjunto como confirmado. Si las predicciones no son correctas, comparar explicaciones alternativas que permitan dar cuenta de lo que ha fallado.

Dado que los economistas trabajan con fenómenos complejos en los cuales se requiere formular muchas simplificaciones y donde muchas interferencias pueden aparecer, parece poco racional descartar una hipótesis debido a una predicción incumplida. Frente a una aparente disconfirmación el economista aplica lo que Hausman denomina el principio del eslabón más débil' : cuando una conclusión falsa depende de un número de premisas inciertas atribuya el error a la más incierta de éstas. 
Los economistas, enfrentados con serias dificultades para contrastar sus teorías -concluye Hausman- confían en las implicancias que surgen de los axiomas de la teoría más allá de los resultados negativos que la contrastación empírica pueda arrojar. Difícilmente una teoría sea dejada de lado debido a un resultado aparentemente disconfirmatorio.

Es que en economía no existen, en general, experimentos decisivos. Dado un resultado econométrico, por ejemplo, basta en muchos casos la inclusión de alguna otra variable, una ligera modificación de los supuestos del modelo o del procedimiento de estimación para obtener resultados distintos y aún contrarios. Los ejemplos abundan al respecto en la literatura. No importa cuán sofisticada sea la técnica econométrica utilizada y cuán amplio y detallado sea el conjunto de datos empleado muy pocas relaciones robustas pueden obtenerse.

McCloskey (1985) argumenta en la misma dirección. En realidad, dice, los economistas -al igual que sus colegas de las otras ciencias- se preocupan por persuadir a sus colegas. La persuasión en economía, sin embargo, no depende tanto de predicciones exitosas como de elementos tales como: elegancia matemática, argumentos por analogía, simetría, etc. Las proposiciones empíricas acerca de la economía real no ocupan un lugar central como ocurre en las ciencias naturales (Bloor-Bloor, 1993).

Como señalara Blaug (1992, p.259), "(los economistas neoclásicos) predican la importancia de someter las teorías a contrastación empírica, pero raramente cumplen con los cánones metodológicos que declaman. La elegancia analítica, la economía de instrumentos teóricos y el más amplio alcance obtenido gracias a heroicas simplificaciones han sido premiados por sobre la predictibilidad y la significación para cuestiones de política".

Mientras un físico o un químico, tras formular una determinada conjetura, puede dirigirse al laboratorio y llevar a cabo un experimento que permita confirmar o disconfirmar aquélla, nada parecido ocurre en economía. Aquí es muy difícil llevar a cabo experimentos y menos aún puede hablarse de un experimento decisivo. Ello pone de manifiesto el craso error que cometen aquellos economistas que equiparan el análisis económico con la física

Es que la dificultad esencial que enfrenta la economía -como el resto de las ciencias sociales- es la imposibilidad, en la gran mayoría de los casos, de efectuar experimentos controlados. Y en el caso de experimentos no controlados, en los que no se tiene dominio alguno sobre las condiciones bajo las cuales el mismo tiene lugar, siempre existe un amplio margen para aducir que la discrepancia entre la predicción y los datos debe atribuirse a que alguna de las circunstancias bajo las cuales la hipótesis es válida no se verificaron. No existe, por tanto, ningún experimento decisivo que permita refutar una teoría.

Más aún, como señala Jon Elster (2009), ninguno de los economistas que ha recibido un Premio Nobel lo ha sido por haber formulado predicciones empíricas que han sido confirmadas. Todo lo contrario sucede en el campo de la física.

En economía, estamos en una situación equivalente a la que ocurriría si los instrumentos de observación y medición disponibles no permitieran discernir si un cisne es blanco o es 
gris. En tal caso pese a que la proposición "todos los cisnes son blancos" es falseable no sería susceptible de ser falseada.

\section{3.- LA REFUTACIÓN EN ECONOMÍA}

¿Quiere eso decir que en economía las teorías nunca se refutan? No, pero la refutación no surge usualmente por medio de los mecanismos de verificación empírica que se estudian en los cursos de estadística y econometría sino por lo que yo llamo "grandes experimentos sociales". Son los "grandes eventos" que menciona Tobin (1996) los que desacreditan determinadas ideas y promueven su sustitución por otras nuevas.

La Gran Depresión de los años 30, por ejemplo, desacreditó la idea que el pleno empleo de los recursos podía alcanzarse en forma automática. Ningún economista razonable pone hoy en duda en Estados Unidos, por ejemplo, el rol de la política monetaria y de la Reserva Federal en la estabilización del ciclo económico.

Durante muchos años se discutió acerca del papel de la política monetaria en la generación de la inflación. Pero los procesos de alta inflación de los años 70 y especialmente los casos de hiperinflación, como el de la Argentina, despejaron dudas acerca de la necesaria participación del componente monetario en dichos procesos y del rol de la política monetaria en su corrección.

Este es el tipo de refutación que históricamente ha operado en economía. Pero, salvo este tipo de casos extremos, es válida la reflexión de Hahn (1987, p.110) acerca de que "no es fácil encontrar una proposición en Economía que todo economista razonable concuerde en que ha sido falseada decisivamente por la evidencia".

Todo esto puede resultar sorprendente para quienes identifican la actividad científica con una secuencia consistente en la formulación de hipótesis, contrastación empírica de sus implicaciones y descarte de las que resulten refutadas. Sin embargo, Kuhn (1962, pp. 128/9) señala que los científicos, ni siquiera cuando se enfrentan a anomalías graves y prolongadas renuncian al paradigma que los ha conducido a la crisis. Por el contrario, inventan numerosas articulaciones y modificaciones ad hoc de su teoría para eliminar cualquier conflicto aparente. "Para que una anomalía provoque crisis debe ser algo más que una simple anomalía" (ibíd., p.135). Además, suele transcurrir "un periodo considerable entre la primera percepción del trastorno y el surgimiento de un nuevo paradigma" (ibíd., pp.141/142).

Para que un nuevo paradigma se imponga tiene que demostrar no sólo su habilidad para resolver los problemas que el antiguo se mostraba incapaz de solucionar sino que, además, debe evidenciar que puede dar respuesta a todas las cuestiones que éste parecía resolver.

Debe tenerse en cuenta, además, que los científicos en general y los economistas en particular son seres humanos que tratan de preservar el capital humano acumulado a lo largo de 
años, por lo que tienen una natural resistencia a admitir que se requiere un cambio de paradigma que puede tornar obsoletos muchos de los conocimientos y habilidades adquiridos. No es de extrañar que los nuevos paradigmas sean introducidos por jóvenes que no están aún demasiado "comprometidos con las reglas tradicionales de la ciencia normal debido a que tienen poca práctica anterior” (ibíd., p.147).

\section{4.- LA PREDICCIÓN EN ECONOMÍA}

Friedman, en su famoso texto de 1953, instaló la predicción como criterio de demarcación en Economía. La teoría debe ser evaluada por su capacidad predictiva para la clase de fenómenos que intenta explicar, señaló. "La única prueba relevante de la validez de una hipótesis es la comparación de sus pronósticos con la experiencia" (Friedman 1953, p.50. Énfasis mío).

Sin embargo, la predicción en nuestra disciplina presenta particulares dificultades. Difícilmente pueda alcanzarse un nivel de precisión cercano al de la física, la medicina o aún la meteorología. En realidad, es mucho más defendible la predicción cualitativa -el signo del cambio- que la cuantitativa -su magnitud. Pese al uso de cada vez más sofisticadas técnicas econométricas y de más versátiles programas de computación el impacto en el mejoramiento de la capacidad predictiva ha sido escaso.

Ello ha llevado a McCloskey a concluir que la predicción es imposible en economía. Citando a von Mises, señala que la predicción del futuro económico se encuentra más allá de la capacidad de cualquier mortal (McCloskey, 1998, p.151). En promedio, la industria de producción de pronósticos obtiene sólo beneficios normales, apunta.

\section{5.- EL USO DE LA MATEMÁTICA EN ECONOMÍA}

Las críticas a la teoría neoclásica suelen ir acompañadas de la impugnación al uso de la matemática en Economía.

Este es un tema que ya se discutió en los '40 y '5o pero que reaparece periódicamente.

Sobre el uso de las herramientas matemáticas, Williamson (2000, p.605) advierte sobre la posibilidad de que aspectos centrales de la teoría sean dejados de lado u oscurecidos por su traducción al lenguaje matemático.

En el mismo sentido, Debreu (1991, pp.4/5) reconoce que el uso de la matemática impone determinadas condiciones. La propia elección de las preguntas a responder puede estar influenciada por el bagaje matemático del economista. La economía puede llegar a jugar 
-advierte- un papel secundario en dicha elección. La matemática es extremadamente demandante: exige incesantemente supuestos más y más débiles para obtener resultados más generales.

La posibilidad de la modelización matemática exige, en muchos casos, la adopción de "supuestos heroicos" que permiten salvar el modelo pero a costa de echar por la borda el contacto de los supuestos con la realidad. Ello hace que en muchos casos -más de los que sería aceptablese concluya con modelos que cautivan por la elegancia y belleza estética de sus demostraciones pero cuyas conclusiones tienen poco o nada que ver con la realidad. Esto no sería tan grave si no fueran luego utilizados por funcionarios o consejeros poco ilustrados que olvidan el costo pagado para alcanzar dichas conclusiones.

Obviamente, la ventaja que aporta la matematización de la economía es evitar errores lógicos. Dadas las dificultades que enfrentan los experimentos en esta disciplina, la teoría económica depende críticamente del discurso lógico. En física, la observación empírica y los resultados experimentales permiten controlar las construcciones teóricas; ello ha permitido ocasionalmente emplear razonamientos que se apartan conscientemente de los cánones de la deducción matemática. Ello no es admisible en la teoría económica, dado que la consistencia interna es la única garantía de rigurosidad.

La matemática es un lenguaje, como Samuelson recordaba en sus Fundamentos, popularizando la afirmación de Gibbs. No es más ni menos que un lenguaje. No hay razones para sostener que sea el lenguaje de la Economía. Si la consistencia lógica puede garantizarse sin el uso de la Matemática, ¿̇cuál es la necesidad de utilizarla? Por otro lado, si sólo ella nos permite arribar a conclusiones que no pueden alcanzarse empleando solamente el razonamiento lógico, ¿por qué no emplearla?

En tal sentido debe reconocerse que han sido textos no matemáticos los que mayor influencia han ejercido en la profesión. Valga como ejemplo la obra sobre historia monetaria de Friedman y Schwartz (1963) que hizo mucho más por la difusión de las ideas del monetarismo que muchos sofisticados modelos econométricos, (Summers, 1991) o el caso de la Teoría General de Keynes, para citar dos de los textos económicos de mayor influencia en el siglo XX.

\section{6.- ECONOMÍA POSITIVA Y ECONOMÍA NORMATIVA}

David Hume fue el primero en distinguir entre el "ser" y el "deber ser". John Neville Keynes introdujo la distinción entre economía positiva, economía normativa y el arte de la economía. Mientras la economía positiva se ocupa de lo que es y la normativa de lo que debe ser, el arte de la economía es el puente entre ambas, utilizando los conocimientos que aporta la primera para alcanzar las metas que determina la segunda. 
Se sostiene habitualmente que la economía positiva es una ciencia tan objetiva como cualquiera de las ciencias físicas y que sobre sus aseveraciones no debería haber discrepancias entre los economistas. Las mismas sólo tendrían sentido en el caso de la economía normativa, afectada por juicios de valor.

Sin embargo, se pasa por alto una cuestión metodológica. La economía positiva utiliza lo que Hausman llama el método deductivo. Se parte de determinados supuestos básicos y generalizaciones y, a partir de ellos, se obtienen predicciones por vía deductiva. Por tanto, la economía positiva trata, no de lo que es, sino de lo que sería si el mundo se ajustara a los supuestos de la teoría económica. En la medida en que se respeten las reglas de la lógica deductiva, nadie puede discutir las inferencias obtenidas. Pero variando los supuestos se llega por la misma vía a conclusiones diferentes y aún opuestas.

Por ejemplo, un supuesto básico de la economía neoclásica es la existencia de racionalidad ilimitada en los agentes. Las conclusiones son, por ende, válidas para un mundo con agentes ilimitadamente racionales; para el mundo que sería bajo tal condición. En cambio, en las ciencias naturales, ningún supuesto se formula respecto a cómo deciden las partículas su comportamiento. Se describe el comportamiento observado de las mismas.

Lo que nos dice la economía neoclásica es cómo sería el mundo si -y sólo si- los consumidores fueran todos maximizadores de utilidad o las empresas maximizadoras de beneficios. $\mathrm{O}$ si no lo fueran pero se comportaran como tales.

Es por ello que hay muchos que interpretan la teoría económica como normativa: nos dice lo que debería ser para que la conducta de los agentes sea racional, lo que las firmas deberían hacer para maximizar sus beneficios, etcétera.

Ello hace que aún la propia economía positiva sea objeto de discusión y disenso, particularmente en cuanto a la relevancia, pertinencia y utilidad de los supuestos. Obviamente, si existiera un experimento decisivo que nos posibilitara discriminar tajantemente entre teorías la dificultad sería mínima. Una vez más, es su carencia en la mayoría de los casos lo que da un carácter distintivo a la economía y al resto de las ciencias sociales, imposibilitando obtener refutaciones concluyentes.

Por tanto, la economía positiva está lejos de ser un conjunto de conocimientos sistematizados acerca de porciones de la realidad, como suele encontrarse en las ciencias positivas de la naturaleza. Más bien, se trata de un conjunto de teorías y modelos disponibles en una gran "caja de herramientas" para ser utilizados según sea el caso bajo análisis y el buen sentido del analista.

$\mathrm{Y}$ esto es así por las particulares condiciones a que se ve sujeta la economía -al igual que el resto de las ciencias sociales-: sus agentes son seres humanos que toman decisiones, interactúa una multiplicidad de variables y no existen -para la gran mayoría de los fenómenosexperimentos controlados.

En cuanto a la economía normativa, para Keynes (padre) debía examinar los criterios de lo que debería ser, esto es de lo que se considera deseable. Finalmente, el arte de la economía 
debía ocuparse de utilizar las herramientas y conocimientos provistos por la economía positiva para alcanzar las metas identificadas por la economía normativa.

Sin embargo, esta clasificación tricotómica no prosperó. En general, se distingue entre economía positiva y normativa, entendiéndose que el análisis de metas y de medios para lograrlas es parte del capítulo normativo de la Economía.

Sea que se adopte la división dicotómica o tricotómica, lo cierto es que tradicionalmente se ha visto como una de las misiones de la economía positiva la de ser útil para orientar la política económica. Y aquí es donde surge la cuestión acerca de en qué momento deben introducirse las dimensiones no económicas -políticas, sociológicas, psicológicas, etc.

Hausman critica a la teoría económica el dejar fuera del análisis los factores causales no económicos, constituyendo por ello lo que él denomina una ciencia "separada". Sin embargo, no parece ni tan sencilla ni tan necesaria la incorporación de factores no económicos en el campo de la teoría económica. Pero, en cambio, parece impensable formular política económica ignorando la relevancia de las variables no económicas. Una cosa es tener en cuenta, como estrategia metodológica, sólo el campo de los factores económicos en el marco de la teoría y otra muy diferente ignorar la influencia de los otros factores causales como si no existieran cuando se pretende operar sobre la realidad. Si se los ignora, la dura realidad se ocupará de poner de relieve que aquéllos siguen presentes.

Sucede al respecto algo similar a lo que suele acontecer en general respecto a los supuestos utilizados en la teoría económica. Una cosa es usarlos para simplificar el análisis y otra considerarlos como atributo de la realidad analizada. Por ejemplo, es común advertir que del supuesto teórico de flexibilidad simétrica de precios se pasa luego a razonar como si ésa fuera una condición de la realidad empírica a la que se pretenden aplicar determinadas políticas. Otro ejemplo es identificar el supuesto sobre la inexistencia de restricción de capital con dicha condición como un atributo necesario de la realidad económica; y así existe enorme cantidad de casos similares.

Del mismo modo, de la no consideración de los factores causales no económicos en la teoría se suele pasar a no considerarlos en la formulación de políticas económicas con los lamentables resultados muchas veces vistos. Aunque el decisor ignore las variables no económicas ellas siguen ahí presentes ejerciendo toda su influencia.

\section{7.- COMPLEJIDAD Y ECONOMÍA}

Nadie puede dudar que la economía constituye un sistema sumamente complejo. El enfoque tradicional ha sido utilizar la abstracción de modo de reducir las complejidades de la economía real a reglas y conductas simples, dejando de lado todo lo que pueda resultar secundario. 
Sin embargo, una alternativa es estudiar los sistemas económicos utilizando el enfoque de la complejidad hoy en boga en otras ciencias. Su punto de partida es sostener que el reduccionismo no es adecuado para estudiar sistemas como el económico en el que interactúan muchas partes para generar un comportamiento global, el cual puede ir mucho más allá de lo que surge del desenvolvimiento de sus partes constitutivas. De la interacción pueden surgir nuevas conductas o fenómenos; el estudio de estos comportamientos o fenómenos emergentes es el objetivo del enfoque de la complejidad.

La simulación mediante el uso de computadoras y el empleo de métodos experimentales para determinar por la vía inductiva posibles resultados y rangos de soluciones son las herramientas que este enfoque emplea.

Brian Arthur (2014) sostiene que "la economía de la complejidad nació en 1987 cuando la ahora famosa conferencia de científicos y economistas convocada por el físico Philip Anderson y el economista Kenneth Arrow se reunieron para analizar la economía como un sistema complejo y evolutivo".

Hay muchas definiciones de "complejidad"; el físico Seth Miller recopiló un total de 45, lo cual llevó al periodista científico Horgan (1995) a quejarse de que "hemos ido de la complejidad a la perplejidad”.

Rosser (2004) compiló en tres volúmenes gran cantidad de trabajos en que se aplica el enfoque de la complejidad a la Economía.

Rosser brinda una definición amplia, siguiendo a Richard H. Day (1994): un sistema dinámico es complejo si endógenamente no tiende asintóticamente hacia un punto fijo o un ciclo límite pero tampoco explota.

Mientras los sistemas lineales o bien convergen a un punto fijo o siguen un sendero explosivo los sistemas dinámicos no lineales pueden tender hacia otros tipos de atractores como un ciclo límite o atractores periódicos, cuasi-periódicos o caóticos.

El estudio de sistemas dinámicos no lineales constituye uno de los capítulos principales de la economía de la complejidad. Modelar las fluctuaciones económicas y el comportamiento de los mercados financieros son dos campos importantes aunque no exclusivos de aplicación de este enfoque.

\section{8.- CONCLUSIONES}

El trabajo analiza algunas cuestiones atinentes a la metodología económica.

El hecho que el objeto epistemológico de la economía sea marcadamente diferente del de las ciencias naturales induce a pensar que el enfoque metodológico también debería serlo. 
La principal limitación que enfrentan las ciencias sociales -y que las diferencia de las naturales- es la dificultad para llevar a cabo experimentos controlados. Ello hace extremadamente dificultosa la verificación de las predicciones y, más aún, la refutación de cualquier hipótesis.

En todo caso, la refutación surge por la vía de lo que hemos denominado los "grandes experimentos sociales", que desacreditan y sepultan determinadas ideas.

Si bien la economía formula teorías falseables, las mismas, en su mayoría, no pueden ser falseadas. Los economistas, enfrentados con serias dificultades para contrastar sus teorías confían en las implicancias que surgen de los axiomas de la teoría más allá de los resultados negativos que la contrastación empírica pueda arrojar.

La matematización de la economía permite evitar errores lógicos. Pero hay que recordar que la matemática no es más ni menos que un lenguaje. No hay razones para sostener que sea el lenguaje de la Economía. Si la consistencia lógica puede garantizarse sin el uso de la Matemática, ¿̇cuál es la necesidad de utilizarla? Por otro lado, si sólo ella nos permite arribar a conclusiones que no pueden alcanzarse empleando solamente el razonamiento lógico, ¿̇por qué no emplearla?

La economía positiva no trata de lo que es sino de lo que sería si el mundo se ajustara a los supuestos de la teoría económica. Esto debe recordarse al momento de aplicar sus conclusiones a la realidad: los supuestos de la teoría no necesariamente son una condición de la realidad empírica.

Finalmente, el trabajo destaca las posibilidades que ofrece aplicar el enfoque de complejidad a la economía. Se señala que el estudio de sistemas dinámicos no lineales permite modelar las fluctuaciones económicas y el comportamiento de los mercados financieros.

\section{REFERENCIAS BIBLIOGRÁFICAS}

Blaug, M. (1980, 1992). The Methodology of Economics. Cambridge University Press, New York.

Bloor, M.-Bloor T.(1993). How economists modify propositions, en Henderson, W. et al. (ed.), Economics and Language, Routledge, Londres.

Debreu, G. (1991). "The Mathematization of Economic Theory". American Economic Review, March, 1-7.

Friedman, M. (1953). La metodología de la Economía Positiva. Reproducido en F. Hahn y M. Hollis (comp.) (1986), Filosofía y teoría económica, Fondo de Cultura Económica, México. 
Friedman, M.-Schwartz A.J. (1963). Monetary Trends in the United States and the United Kingdom: Their Relation to Income, Prices and Interest Rates, 1867-1975, University of Chicago Press, Chicago.

Hahn, F. (1987). "Review of D. McCloskey, The rhetoric of economics". Journal of Economic Literature, 25 (1), 110-111.

Hausman, D.M.(1992). The inexact and separate science of economics. Cambridge University Press, Cambridge.

Horgan J. (1995) “From complexity to perplexity” Scientific American 272(6), pp.104-109

Kuhn, T.S. (1962). La estructura de las revoluciones científicas. Fondo de Cultura Económica, México.

McCloskey, D. N.(1985, 1998). The Rhetoric of Economics. University of Wisconsin Press, Madison.

McCloskey, D. N.(1991). "Economic science: a search through the hyperspace of assumptions?, Methodus 3(1), 6-16.

Rosser J. B. (2004) Complexity in economics, Edward Elgar, Cheltenham Northampton.

Summers, L. (1991). "The Scientific Illusion in Empirical Macroeconomics", Scandinavian Journal of Economics, vol. 93, March, 129-148.

Tobin, J. (1996). Full Employment and Growth: Further Keynesian Essays on Policy, Edward Elgar, Cheltenham.

\section{COMO CITAR ESTE ARTÍCULO}

BEKKER, Víctor A. Algunas cuestiones metodológicas en el análisis económico. Revista de la Facultad de Ciencias Económicas - UNNE, Argentina. Volumen 22 Núm. 1, enero-junio 2019, ISSN 1668 - 6365. Págs. 15 - 23. DOI: http://dx.doi.org/10.30972/rfce.2213946 


\section{CURRICULUM VITAE}

\section{Víctor A. Beker}

Director del Centro de Estudios de la Nueva Economía de la Universidad de Belgrano y Profesor Titular Consulto de la Universidad de Buenos Aires. Ex Director Nacional de Estadísticas de las Actividades Productivas del INDEC. Fue profesor invitado con rango de Catedrático en la Universidad de Salamanca e Investigador Invitado en el C.V. Starr Centre for Applied Economics de la New York University. Premio Salvador Aisenstein del Colegio de Graduados en Ciencias Económicas y Premio Academia Nacional de Ciencias de Buenos Aires. Autor de libros y artículos en la especialidad.

victor.beker@ub.edu.ar 